\title{
Rethinking the musical ensemble: A model for collaborative learning in higher education music technology
}

Oded Ben-Tal, Kingston University London

\author{
Diana Salazar, City University London
}

\begin{abstract}
Kudac (Kingston University Digital Arts Collective) is an electronic improvisation ensemble that brings staff and students together for weekly musicking with technology - incorporating resources ranging from conventional instruments, to computers, to hacked circuit boards. A central element of the ensemble from its inception has been its democratic approach - staff and students explore the musical possibilities and challenges together and gradually mould their practice through a free exchange. In this article we consider the contribution of this ensemble in several overlapping domains: in relation to the individual students, in the context of a higher education music department, and at the intersection of research and teaching. We first survey the structure and activities of the ensemble, contextualizing this with reference to existing research in the fields of laptop performance, free improvisation and musical identity formation. We use this as a platform for tracing how such an ensemble may aid the social construction and shaping of creative identities at both an individual and collective level. We then examine the opportunities and challenges for a music department hosting such an ensemble before highlighting areas for future study.
\end{abstract}

\section{Keywords}

Creativity 
Creative collaboration

Free improvisation

Higher education

Laptop ensembles

Laptop performance

Music technology

\section{Context: The growth of laptop ensembles}

The past decade has seen tremendous growth in laptop orchestras, spurred by the proliferation of lower cost computing and improvements in laptop processing power. In recent years the tools available to digital performers have expanded exponentially to include low-cost controllers, sensors and circuits and, more recently, the possibilities of live coding and networked performance. Spearheaded by the development of early orchestras such as PLOrk (see Trueman 2007), digital ensembles have become a standard fixture in many university music departments, particularly in Europe and North America. A recent study by Knotts and Collins (2014) collated data on no less than 160 laptop orchestras worldwide (though not all were affiliated with academic institutions). Of course, many more derivative ensembles exist, for instance those combining acoustic instruments and laptops, those utilizing a broader range of technologies (such as circuit bending), cross-disciplinary collectives and other hybrid ensembles (Webb and Seddon 2012). 
In Britain there is almost certainly a correlation between the growth of these ensembles and the exponential growth of music technology undergraduate degrees over the past ten years. As Boehm (2007) points out, this music technology category covers a wide range of degree programmes each combining elements from music, technology, science and design. ${ }^{1}$ One result of this expansion in music technology provision is a growing body of students whose principal study may not necessarily be a 'conventional' instrument such as flute or electric guitar, but instead a computer (in the non-performative sense). As existing groups such as PLOrk, Benoît and the Mandelbrots, and Music Hackspace gain increased exposure and publications illuminate the pedagogical benefits of such groups, many more academics (and students) may consider establishing similar groups in their own departments. However the overwhelming array of possible technologies, approaches and skills involved creates a complex landscape that warrants greater analysis and conceptualization.

Kudac (Kingston University Digital Arts Collective) is an extra-curricular ensemble run weekly during term time. ${ }^{2}$ It was established in 2011 by four staff members at the music department of Kingston University (Drs Diana Salazar, Louise Harris, John Ferguson, Oded Ben-Tal) with the explicit aim of creating a shared space linking practice-based research and teaching. It is open to all students, regardless of musical or technical experience, at undergraduate and postgraduate levels on any course, although it attracts particular interest from students enrolled on the BMus Creative Music Technologies programme. From its inception, the creative exploration of sound (in its broadest sense) has been central to Kudac and is the glue that connected four staff composers coming together from different backgrounds and with divergent ideas 
about music and composition. These composers have employed a variety of techniques and tools to facilitate meaningful engagement with music technology.

\section{Existing research}

Recent studies have unpacked dimensions of these electronic ensembles, foregrounding aspects such as their capacity to develop transferable skills (Mudd 2012), to encourage critical reflection (Tsabary 2014), as well as discussing performance strategies and models for the musical and social interactions within these types of ensemble (Albert 2012; Booth and Gurevich 2012). Blain (2013) also problematizes the nature of performance when working collaboratively with laptops as instruments. The issues raised in these studies converge with broader areas of research in music technology such as the development of new interfaces for musical expression and the performance practice of electronic music. ${ }^{3}$ Given the fascinating array of ensembles emerging, it is no surprise that laptop ensembles have generated a melting pot of discussion and debate straddling the areas of performance, technology and pedagogy.

Simultaneously, rich discourses are emerging on the socially constructed nature of musical creativity and musical identity. Notable contributions to these fields include insights by David Hargreaves (in Hargreaves et al. 2002, 2012) and Pamela Burnard (2012a, 2012b), who both highlight the significance of social interactions (on micro and macro scales, and in real and virtual domains) in shaping the creative identity of individuals.

This article will begin to draw connections between the technological tools and the social frameworks in these emerging digital music collectives. We present a case study of an ensemble and examine its form in order to better understand the creative 
currents that flow inside and around its creative ecology. By surveying a range of musical motivations and techniques we will trace the social formation of creative, and particularly compositional identity at both individual and collective levels. We will examine how particular compositional and improvisational approaches in the context of digital technology and ensemble practice serve to reinforce the individual student's compositional practice. What steps are required in order to move from collaboration in principle, to meaningful creative performance? And what challenges does this present to mentors and the host department? We examine the opportunities and challenges presented by the Kudac model, before highlighting areas for future study.

\section{Isolated learning experiences}

The pedagogical benefits of collaborative learning in higher education are clear (see Orr 2010; Hakkarainen 2013), but much student learning in higher education today still involves isolated learning experiences (Tinto 2003; Cain 2004; Salazar 2010; Christophersen 2013) and this is particularly the case for music technology students, where the computer workstation is often the focal point for skills development. Regardless of a student's choice of musical style, creative engagement in music technology is often dominated by independent work (for instance composition, mixing, or mastering) in the studio, a tendency recognized by Savage (2012). Even a group recording session may position the music technology student behind the glass of the control room, creating a physical and psychological partition between those who perform, and those who operate technology. And despite a collaborative ethos underpinning many types of popular music production, the role titles of 'music producer' and 'artist' assist in demarcating the technologists from performers. 
As such, music technologists tend to stand apart from their peers in music, drama and dance where group interaction is ingrained in performance traditions. In music, the opportunity to make music in an ensemble is widely recognized as important in developing students' understanding of music, music making and musical culture, as well as vital communication skills (Kokotsaki and Hallam 2007; Harrison et al. 2013). Yet many of the students who choose to study music technology may arrive at university without experience in conventional instrumental performance, instead identifying the computer as their primary instrument, and often in a non-performative sense. As a result, these students may struggle to identify themselves as 'performers' and may feel alienated or ineligible to participate. One might go as far as to trace an emerging bifurcation, to paraphrase Savage; those who perform, and those who do music tech.

\section{Composing in the box}

Engaging with music creation primarily through a computer screen also shapes students' conceptual framework. Music software packages, such as notation editors and audio sequencers, lead to different affordances for thinking in sound (Gall and Breeze 2005; Wiggins 2007). Often students will construct electronic compositions as discrete tracks, conforming to beat grids, which are then layered on top of each other. Each track may then have effects (for instance delays, distortions, reverb, etc.) applied to it. It is not uncommon for the amount of technology used and the type (usually the more expensive the better) to become associated with quality through a kind of fetishization of technological apparatus at the expense of aesthetic considerations. In turn, students may perceive a primary compositional goal as the acquisition of more expensive, cutting edge, and/or coveted and rare technologies (e.g. microphones, plug-ins, analogue synthesizers) which are seen as the principal route to producing 
better music. Curriculum provision and teaching methods need to be adapted to accommodate but also challenge this musical thinking, where compositional process is refracted by the lens of the digital audio workstation.

\section{Musical identities and values}

Many, if not all of the music technology students at Kingston University view themselves as studio producers and non-performers. A number of existing reference points for performance are problematic for a laptop ensemble (see Blain 2013). Students might associate performance with the western classical tradition of mastering a traditional instrument (or the voice), a role with which they are unlikely to identify. In popular music, the rock band has traditionally been situated outside formal education. And the mainstream DJ figure is an individual placed at the epicentre of the music performing a well-defined instrument (traditionally the decks) and exploring an explicit musical framework. One challenge when establishing an experimental digital ensemble is to redefine performance in a way that is meaningful, relevant and engaging for students.

More broadly, in a university environment each student is exposed to competing influences on their identity; perceptions of the external 'music industry', the expectations of the academic institution, and the student's own values and intuition, which have been shaped by a complex network of experiences and creative encounters. Frequently there are tensions between each of these influences, and a further challenge for any ensemble is to illuminate the underlying 'interconnectedness of learning contexts’'(O’Neill 2012) without stifling existing values. 
The result of these factors is that music technology students may feel disconnected from two critical ingredients of musical development - music as a performance activity and the social, collaborative aspects of music as a creative practice (Burnard 2012a). Laptop ensembles create a space where music technology students can engage collaboratively in musicking, a place that 'offers opportunities for meaningful participatory music engagement' (O’Neill 2012) which embeds technology as part of creative practice.

\section{Improvisation as real-time composition}

It is common for laptop ensembles to utilize what Tsabary (2014) terms a 'comprovisational' approach, a blend of composition and improvisation (Dudas 2010; Ben-Tal and Wilkins 2013). Even composed works where participants each utilize the same composer-prescribed software will often utilize a combination of what Nettl, talking about improvised musics, identified as structural markers or signposts (Nettl 1974) and passages of extemporization (see Albert 2012 for examples). This reflects the ongoing evolution of both instruments and ensembles and the associated difficulties in standardizing repertoire. Broadly speaking this is the approach taken by laptop orchestras such as Plork, Slork, or L2Ork. In these ensembles the setup is mostly similar or identical for each player, using a set hardware configuration and usually identical software. As the name laptop orchestra indicates these ensembles aim to update the notion of a music ensemble with clear roles of performer, composer, conductor. In contrast Kudac (and ensembles that took a similar approach such as Huddersfield's HELO) encourages participants to develop their own individual approach utilizing whatever tools they prefer. $^{4}$ By removing many of their usual compositional constraints, students are then challenged to design their own, and in 
doing so, construct a role for themselves as part of the group. Here the roles of performer, composer and conductor become much more fluid, moving away from the power model seen in orchestras and towards the democratic model of many free improvising instrumental collectives.

Rose and MacDonald note improvisation's ‘ubiquitous presence within all forms of music-making' (2012: 187), although the practice of digital improvisation is still being forged and the relevance of improvisation for the studio composer is rarely articulated. In a similar way, Wilson and MacDonald (2012: 559) note the 'universal capacity' of improvisation, 'since anyone who enjoys music can participate in group improvisation to some extent, irrespective of musical training', though one suspects that skill compatibility between participants would play a role. The skills required include perceptive listening to both local sonic context and longer-term musical processes, and the ability to exert control in sound production/manipulation. In more conventional ensembles this latter aspect relies on instrumental skills, supported by the music department through individual tuition. Performing through electronic means adds another dimension to the challenge of group improvisation.

There are significant risks to using a free improvised approach. Musical outcomes are susceptible to a lack of direction, leading to ‘aimless play’ (Mudd 2012) and even chaos. The structuring of material can be particularly problematic, and students may struggle to find a route into understanding the music they create collaboratively. Without sufficiently high levels of listening acuity and critical skills to accompany technical expertise, meaningful real-time expression is impossible. Here the word meaningful is crucial. A complex instrument can only become expressive when mediated by listening and critical insight, and it is at this juncture that the development of collective and individual creative identity is forged. As such the 
pedagogical benefits of free improvisation outweigh the risks, particularly in a longerterm view of musical development beyond the three years spent working towards a degree.

\section{Developing tools for real-time expression}

Utilizing strategies such as graphic scores, game-like performance exercises, and free improvisation can provoke students to think about composition in radically new ways, particularly students whose home territory is studio-based production, working with tracks and audio files in a non-real time capacity. The notion of real time musical expression may be a daunting prospect for new members of the ensemble. While the technology being used is familiar (laptops, midi controllers, pedals), it requires a radically new set of technical and musical skills. The learning curve to achieving a controlled and expressive contribution may elicit some frustration, especially when it requires coding using a graphical programming language such as Pd or Max. In these applications a significant level of choice and responsibility is shifted onto the user (rather than being channelled by the commercial software developer). The starting point is a blank canvas for instrument development, control and interface design, which empowers the user to build their own unique instrument but simultaneously challenges their existing understanding of music software. Compositional options expand exponentially, but the tools to implement these decisions are not provided instantaneously or ready built. In this environment community sharing becomes paramount, with patches built, shared and exchanged not only between ensemble members, but also with a wider online community of forums. By persevering with the challenge, and engaging with the support and ideas of local and global composer communities, students can develop new modes of conceptualizing music and an acute 
understanding of the decision-making processes underpinning digital instrument development.

Satisfying and meaningful participation for electronic performers does require at least a foundation level of acquaintance and understanding of the computing system, both in terms of software and hardware. There is a preconception that only complex systems enable meaningful expression, but in a collective, building simpler instruments may be advantageous in shifting the goals from technical virtuosity to skills of listening, critical reflection and role formation in the ensemble. In this way Hattwick and Wanderley note that technically simpler instruments encourage 'a quality of interaction between performers which highlights the creation and expression of relationships; which may help in the creation of a meaningful performance' (2012: 2). In this collaborative context understanding the technical (programming/hardware), aesthetic (sonic potential) and social (potential musical role in an ensemble) aspects of an instrument all require development in order to achieve mastery. The principal aim for each participant becomes not to build a complex instrument, but an instrument that facilitates meaningful contribution to the ensemble. This requires students to (re)evaluate their tools, to consider what is most appropriate in the ensemble situation, and to develop the listening skills to use the instrument effectively. We stress that the tools built for solo performance or production may not map well onto the needs of group performance and the ensuing process of negotiation between an individual's solo and ensemble practice can help to illuminate the constraints and skills required for collective participation and success. This process of adaptation at an individual level is critical in improving the cohesion of the ensemble.

Booth and Gurevich (2012) argue that one other way to establish common ground and coherence in the digital ensemble is to take a common approach to instrument 
building. This is also conducive to peer learning, by establishing common technical ground between participant's practices. However Kudac occupies the middle ground. Many of the participants do utilize Pd, but in their own way, and augmented by an array of different peripherals. In this way, students can be supported fully in developing their understanding of the software, but by adopting a heterogeneous approach where all tools and technologies are welcomed, students have the freedom to work to their existing strengths and interests and build instruments that are individual to them. The ability to claim ownership over an instrument can kick-start the process of carving out an individual voice in the ensemble. That said, the guided element of rehearsals encourages students to review and expand their sound palette through full navigation of a wide range of compositional parameters and ensemble behaviours. This helps to form a framework of comprovisational possibilities that exhibits parallels with Hattwick and Wanderley's 'collaborative dimension space' (2012).

A standardized setup does confer pragmatic benefits. It simplifies the logistics of maintaining a working system - by comparison Kudac sessions regularly commence with 30 minutes (and up to one hour on bad days) of setting up and troubleshooting. With every participant's setup in constant development it often takes the combined expertise of several people (staff and students) to identify and solve issues. While this activity is not particularly musical it does constitute learning. The orchestral model lowers the entry barriers particularly for less experienced musicians or students for whom music is a secondary field. Learning to execute actions (move this slider, rotate this knob) from score-like instructions is relatively easier to master compared to Kudac's approach that asks students to find their own creative voice. On the other hand, we believe that the latter approach is more attuned with an essential aspect of 
university music learning, namely pushing students to take creative risks and develop their own aesthetic sensibilities by becoming more independent learners.

\section{Dialogue and developing a shared culture}

We have already acknowledged the risk of free improvisation becoming aimless and chaotic. To address this, we embed extensive critical discussion and debate in rehearsals, with gentle steering by staff in order to facilitate productive improvisational approaches and musical development. Dialogue is intentionally framed by aesthetic questions, encompassing the evaluation of compositional material, development and form. Improvisations and exercises are deconstructed and evaluated, new ideas are proposed and debated, and constraints are then shaped in preparation for the following 'experiment' and subsequent repetition of the process.

This type of reflective cycle is not unique to Kudac. Tsabary (2014) notes the benefits of a cyclical process of development and discussion in his ensemble, which provides a platform for students to act as co-researchers. Likewise, Mudd (2012) notes the similarities between this kind of activity and Kolb’s experiential learning cycle, and Booth and Gurevich observe in Birmingham Laptop Ensemble (BiLE) a 'reflexive process, where instruments evolve in response to experiences gained from playing them - a process which shares some similarities with the iterative development of compositions' (2012).

But why spend substantial parts of rehearsal time in conversation rather than practice? Savage proposes that critical reflection and reflective action are essential for achieving a higher level of digital literacy, that of 'digital transformation'. Similarly, O’Neill strongly advocates dialogue: 
Only through critical reflection and dialogue can educators and learners create the conditions and circumstances in which they can search together collaboratively for more comprehensible authentic, and morally appropriate ways of valuing and engaging in musical practices. (O’Neill 2012: 174-75)

So not only does dialogue help at a surface level to initiate members of the group and foster a sense of belonging for individual participants, it also assists in demystifying the 'multiple divergent meanings' and the 'irresolvably ambiguous' nature of free improvisation (Wilson and MacDonald 2012: 565, 568). This forms a platform for the construction of an authentic collective identity for the ensemble based on a shared culture. One could say that free improvisation is enabled by developing this kind of shared culture (Campbell 2009). Through rehearsal and discussions we arrive at a shared aural sensibility and mutual understanding that provides the necessary framework to improvise together (not just simultaneously) in the absence of stylistic rules and conventional formulae.

In Kudac the inclusion of more than one member of staff is particularly conducive to developing student's 'capacity for critical reflective or reflexive self-awareness' (O’Neill 2012: 164) while at the same time enculturating students into a 'practice as research methodology’ (Blain 2013: 200). Exposing students to the practices and ideas of their peers is valuable, but engaging with researchers and their discourses further enriches the dialogue. With each of the four founding composers utilizing different tools and methods (and using Kudac sessions as testing ground for developing these), students are exposed to a range of research questions. Debates between staff 
members, and the creative tension evident in them, make clear to students that there is no definitive correct answer to creative questions, and this lays the foundations for meaningful and productive dialogue. This resonates with O’Neill's (2012) concept of transformative music engagement, which encompasses elements of empowerment, connectedness and emotional engagement. She asserts that her concept moves forward from Wenger's communities of practice (2002) in that it embraces notions of difference, and as such is more likely to engage critical enquiry. In line with the emphasis on exploration and process in many forms of practice-led research 'the disclosure of irregularities or the problematising of situations is more significant than solution-finding' (Brown and Dillon 2012: 92).

Much research in humanities happens behind closed doors and there is no equivalent of the science-lab at undergraduate level for students to experience research in a hands-on capacity. In Kudac staff act as researcher mentors and both staff and students are co-researchers. Researchers each bring their own work-inprogress and allied research questions, which converge with student's own interests and goals to form a melting pot of ideas and enquiry, an 'affinity space' (Gee in O’Neill 2012). The process helps to demystify the concept of practice as research, and begins to bring undergraduate and research cultures closer together. In turn, students are encouraged to apply the practice as research methodology in their own project development.

The dialectical process between students and staff can carve out 'trading zones’. Here, ‘liminal meeting points’ or points of correspondence may be recognized (Blain 2013), but at the same time creative tensions can provoke the renegotiation of musical values and constraints, leading to the emergence of new 'alternative models 
and dimensions' (Savage 2012: 179), both in the music itself and in individuals, who are provoked to reflect upon their musical values and choices:

...perspective transformations occur when individuals change their frames of reference by becoming aware of, and reflecting critically on, their assumptions and beliefs, and consciously making and implementing plans that bring about new ways of defining their understanding. (O’Neill 2012: 177)

Dialogue becomes essential as a tool not simply for communicating software techniques and establishing strong social bonding between participants, but it helps participants to draw links between the various skills involved in creative participation. These skills can be paralleled with Brown and Dillon's five modes of compositional engagement; attending, evaluating, directing, exploring and embodying (2012). Through dialogue, participants are encouraged to engage fully with the compositional skills matrix, to situate and evaluate their values through critical enquiry, and in turn shape their own creative identity.

But dialogue does not only exist in spoken form. Part of the process of establishing a Kudac culture is about learning the individual's musical identity (or sonic proclivity). Since we are connected into one sound system there is a dislocation between where the player sits and where their sound emanates (though in rehearsals we use a multichannel system to spread the sounds to help us identify individual voices within the ensemble). And while individual setups are in almost constant flux we eventually learn to identify each other's sounds (at least most of the time) and be 
able to respond more quickly to changes or musical ideas thrown into the mix. This enables episodes of musical dialogue between members (usually two) that happen during a piece and serve as momentary anchor within a fluid, textural musical flow.

\section{A framework for shaping compositional identity}

In her interviews with composers Burnard (2012b: 134) has identified a dynamic relationship between the creative 'self', and externally facing social practices. It is clear that creativity, including compositional creativity, is shaped by 'shared knowledge practices cultivated by [...] knowledge communities' (Hakkarainen 2012: 5). The creative identity of an individual and their social experiences of music are tightly intertwined (see Hargreaves et al. 2012) and learning contexts themselves display interconnectedness (O’Neill 2012). In order to understand the role that Kudac performs in shaping an individual's identity, it is useful to deconstruct the ecology of creative values, skills and ideas in which it is situated. This extends beyond face-toface rehearsals and even the University itself, incorporating a complex range of personal, social and cultural contexts that feed into one's compositional practice (Brown and Dillon 2012).

Each student is exposed to competing influences on their identity; the pull of a future career in the music industry, the demands of an academic environment, and their own evolving sense of musical self. It is important that one does not stifle another - in Kudac the values of one researcher/mentor is no more 'correct' than another. The confluence of creative values, and indeed even the tensions between them, may be productive in exposing students to new ideas, and prompting them to reconsider their compositional voice. 
In Kudac students are encouraged to bring their existing knowledge, interests, and skills, but then carve out a performance presence that blends aspects of the innovator, programmer, producer, improviser, composer, performer and, in some contexts, conductor. The resulting composite role is difficult to define, and impossible to label due to its shifting locus in different ensemble situations. It encompasses crucial aspects of the performer and composer, but is much more complex and fluid (Booth and Gurevich 2012). This kind of role formation can be transformational. In challenging students to reposition themselves there is a disruption of traditional practice, and a reshaping of the student's creative identity. Students are encouraged to explore new tools, new sounds, and new notions of creative practice, all of which can transcend the ensemble situation. The diagram below maps how Kudac informs and encourages socially constructed creativities at collective and individual levels, in faceto-face and virtual contexts: 


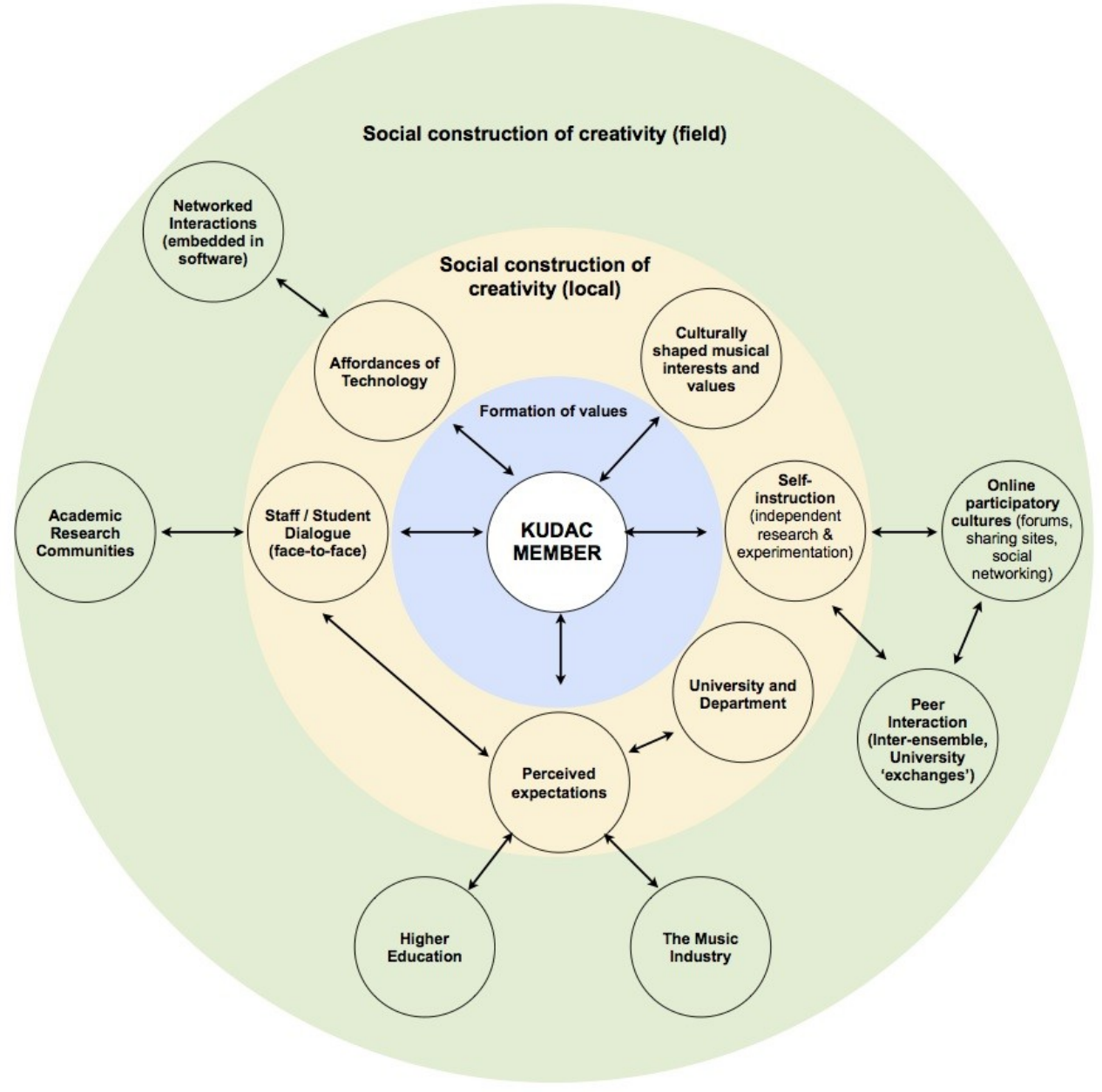

Figure 1: Creative practice occurs within a complex network with individual and social dimensions.

The practice of composition, even in the broadest digital comprovisational sense, is enabled via the process of designing constraints. In the digital domain these constraints extend beyond direct musical parameters and may be embedded at many levels; in the choice and use of hardware, code, peripheral sensors, movement, behaviour, or samples, etc. At the same time these parameters exist in dynamic social frameworks. For instance, the code may be developed in conjunction with a user online forum. The approach to performance motions or behaviour may be informed 
by an artist on YouTube. The sound material may be derived from a life-long passion for drum and bass. Kudac encourages the active and reflexive design of constraints, drawing upon a wide compositional language and a research-informed methodology. Skills from outside the ensemble (i.e. 'field interactions') feed into local ensemble development, which then trickles back into the field communities via the individual's interactions. These transactions are critical, but require framing and direction in order to be most valuable.

\section{Some practical considerations}

In addition to providing a collaborative space for music technology students, the ensemble delivers many benefits to the wider music department. It acts as a vibrant and alternative addition to its portfolio of more traditional ensembles. The inclusive nature of the group ensures that members are welcome from non-music technology degrees at University. This provides a platform ripe for inter-departmental collaboration, an aspect that could be developed further. (Collabhub at the University of Huddersfield is a leading example of large-scale cross-disciplinary collaboration between students from different subject areas of a university, and between students and industry professionals.) At Kingston, students on the most relevant degrees (computing, digital media) are based at a different site from music which means only a handful attended some sessions. However the focus on musical development does present an extra challenge to non-musicians, who may perceive their (lack of) musical skills as one barrier to investing in attending the ensemble regularly.

There are numerous other challenges presented when running this type of collective. It is resource intensive, with particular spacing and equipment requirements for 
rehearsals. This is particularly problematic for Kudac due to the conscious decision to use a high-quality audio setup (involving the purchase of external sound cards, use of a digital mixing desk and high-quality loudspeakers). This is quite different to the economizing approach of many other laptop ensembles. The use of open source Pd software does alleviate some issues, as most students own their own laptop, however providing access to additional hardware, such as midi controllers, is helpful for enhancing expression.

Staff coordinating the ensemble contribute their time for rehearsals outside working hours and their level of commitment frequently extends beyond this to booking equipment and spaces, scouting performance opportunities, editing and organizing rehearsal documentation and promoting the group both internally (through e-mails, concerts and recruitment activities) and externally (through an online presence and external engagements). The latter included exchange concerts with other universities which brought staff and students from both institutions together. In both cases (with the University of Manchester the first year and City University London the second), Kingston's contribution to concerts included Kudac performances alongside compositions by staff members. These events served to showcase the music department externally and also allowed students to encounter new approaches to music and technology as practiced in other environments.

Ensembles thrive when trust is established between staff and students (Harrison et al. 2013; O’Neill 2012), and this can only be nurtured over a prolonged period of time. Similarly, ongoing commitment by participants over a number of years allows the ensemble to strengthen and mature, increasing the potential for peer learning to take place between experienced and novice members (Blom and Poole 2004; Daniel 2004; Lebler 2007). However recruitment and continuity can be a challenge. A process of 
destigmatization may be required for incoming students to consider joining, and committing, to an experimental ensemble. Attending the ensemble can shape how a student is perceived by their peers, and this may elicit scepticism and anxiety about unfamiliar approaches. Curiosity and self-motivation need to win over these anxieties for students to attend. Even once students do begin attending it can be difficult to ensure commitment. Although student commitment to extra-curricular and nonassessed activities is a common difficulty, it is possible that attendance issues are more pronounced in music technology students who, for the most part, have not been enculturated into the tradition of attending (and preparing for) weekly performance activities prior to university.

Finally, staffing changes are inevitable and any ensemble relying heavily on one member of staff may find itself in a precarious position. Although a self-sustaining, student-managed group is an ideal, in practice this is extremely difficult to establish due to the aforementioned issues.

\section{Conclusion}

the multitude of ways and radically changing forms that characterise contemporary compositional practice call for a radically revised conception of a multiplicity of musical creativities. (Burnard 2012b: 114)

Kudac provides a platform for the development of real-time compositional skills that can enhance the non real-time practices of digital musicians. The aim is to develop a 
new kind of musicianship in music technology students, one that is not constrained by the guiding affordances of music sequencers (in the form of beat grids or looping tools) or narrow perceptions of the wider musical world vis-à-vis good and bad music. Similar to traditional musicianship training the aim is to develop nuanced and refined listening skills. These, in turn, enhance music making ability by extending the categories available for conceptualizing sound and practice (Brown and Dillon 2012) and enable students to exert better control of sound parameters. Kudac also opens opportunity for collaborative music-making and peer learning that are not available to music tech students with a non-traditional musical background.

In this article we have shown how such a digital ensemble may be valuable in promoting meaningful engagement with creative practice. In particular, we have identified the potential of improvised collaborative practice to challenge students' musical values and shape a new kind of musicianship, one which has the ability to transcend the ensemble and encourage repositioning of a student's own, individual practice as a composer. Our conceptual model of the social currents surrounding this creative ecology begins to map out the links and creative impact which this ensemble may reinforce.

It must be acknowledged that no two digital ensembles are the same, due to vast possibilities in terms of tools, political structure, intentions and situation, and we do not propose that Kudac is a definitive model for higher education. The fact that technologies are so diverse and constantly evolving creates a fluid and dynamic situation for exciting emergent creativities. In such an environment the agnostic approach to the technology tools that epitomizes Kudac could prove useful despite the logistical headaches it entails. Similarly the democratic ethos of the ensemble 
supports the necessary shift in student's view of education towards a learning partnership and increasing independence.

\section{References}

Albert, J. (2012), 'Improvisation as tool and intention: Organizational practices in laptop orchestras and their effect on personal musical approaches', Critical Studies in Improvisation/Études Critiques En Improvisation, 8:1, n.p.

Ben-Tal, O. and Wilkins, C. (2013), 'Improvisation as a creative dialogue', Perspectives of New Music, 51:1, pp. 21-39.

Blain, M. (2013), 'Issues in instrumental design: The ontological problem (opportunity?) of “liveness” for a laptop ensemble', Journal of Music, Technology \& Education, 6:2, pp. 191-206.

Boehm, C. (2007), 'The discipline that never was: Current developments in music technology in higher education in Britain', Journal of Music, Technology \& Education, 1:1, pp. 7-21.

Booth, G. and Gurevich, M. (2012), 'Collaborative composition and socially constructed instruments: Ensemble laptop performance through the lens of ethnography', in G. Essl, B. Gillespie, M. Gurevich, and S. O’Modhrain (eds), Proceedings of the International Conference on New Interfaces for Musical 
Expression, Ann Arbor, Michigan, USA, 21-23 May.

Born, G. (2015), Music, Digitisation, Mediation: Towards Interdisciplinary Music Studies, http://musdig.music.ox.ac.uk/. Accessed 28 August 2014.

Brown, A. R. and Dillon, S. (2012), Meaningful Engagement with Music Composition, Farnham: Ashgate.

Bukvic, I. I., Martin, T., Standley, E. and Matthews, M. (2010), 'Introducing 12ork: Linux laptop orchestra', in K. Beilharz, B. Bongers, A. Johnston, and S. Ferguson (eds), Proceedings of the International Conference on New Interfaces for Musical Expression, Sydney, Australia, 15-18 June, pp. 170-73.

Burnard, P. (2012a), Musical Creativities in Practice, Oxford: Oxford University Press.

(2012b), ‘The practice of diverse compositional creativities', in D. Collins (ed.), The Act of Musical Composition: Studies in the Creative Process, Farnham: Ashgate, pp. 111-38.

Cain, T. (2004), 'Theory, technology and the music curriculum', British Journal of Music Education, 21:02, pp. 215-21.

Campbell, P. S. (2009), 'Learning to improvise music, improvising to learn music', in G. Solis and B. Nettl (eds), Musical Improvisation: Art, Education, and 
Society, Urbana-Champaign: University of Illinois Press, pp. 119-42.

Christophersen, C. (2013), 'Perspectives on the dynamics of power within collaborative learning in higher music education’, in H. Gaunt and $\mathrm{H}$. Westerlund (eds), Collaborative Learning in Higher Music Education, Farnham: Ashgate, pp. 77-86.

Collabhub, ‘Innovation through Collaboration’, http://blogs.hud.ac.uk/services/collabhub/. Accessed 28 August 2014.

Cycling 74 (2015) Max 7 At A Glance, http://cycling74.com/products/max/. Accessed 30 August 2014.

Daniel, R. (2004), 'Peer assessment in musical performance: The development, trial and evaluation of a methodology for the Australian tertiary environment', British Journal of Music Education, 21:01, pp. 89-110.

Dillon, T. (2007), 'Current and future practices: Embedding collaborative music technologies in English secondary schools’, Music Education with Digital Technology, pp. 117-27.

Dudas, R. (2010), ““Comprovisation”: The various facets of composed improvisation within interactive performance systems’, Leonardo Music Journal, 20, pp. 2931. 
Gall, M. and Breeze, N. (2005), 'Music composition lessons: the multimodal affordances of technology', Educational Review, 57:4, pp. 415-33.

Hakkarainen, K. (2013), 'Mapping the research group: Expertise, collective creativity and shared knowledge practices', in H. Gaunt and H. Westerlund (eds), Collaborative Learning in Higher Music Education, Farnham: Ashgate, pp. $13-26$.

Hargreaves, D. J., MacDonald, R. and Miell, D. (2012), 'Musical identities mediate musical development', in G. E. McPherson and G. F. Welch (eds), The Oxford Handbook of Music Education, Volume 1, Oxford: OUP, pp. 125-42.

Hargreaves, D. J., Miell, D., and MacDonald, R. A. R. (2002), 'What are musical identities, and why are they important?', in R. A. R. MacDonald, D. J. Hargreaves, and D. Miell (eds), Musical Identities, Oxford: OUP, pp. 1-20.

Harrison, S. D., Lebler, D., Carey, G., Hitchcock, M. and O’Bryan, J. (2013), ‘Making music or gaining grades? Assessment practices in tertiary music ensembles', British Journal of Music Education, 30:01, pp. 27-42.

Hattwick, I. and Wanderley, M. M. (2012), ‘A dimension space for evaluating collaborative musical performance systems', in G. Essl, B. Gillespie, M. Gurevich, and S. O’Modhrain (eds), Proceedings of the International Conference on New Interfaces for Musical Expression, Ann Arbor, Michigan, 
USA, 21-23 May.

Hewitt, S., Tremblay, P. A., Freeman, S. and Booth, G. (2010), 'HELO: The laptop ensemble as an incubator for individual laptop performance practices’, in Proceedings of the International Computer Music Conference, New York, 1-5 June.

Input Devices and Music Interaction Laboratory at McGill University (2014), The McGill Digital Orchestra, http://www.idmil.org/projects/digital orchestra. Accessed 28 August 2014.

Kingston University Digital Arts Ensemble (2015), KUDAC Soundcloud, https://soundcloud.com/kudac. Accessed 29 January 2015.

Knotts, S. and Collins, N. (2014), 'The politics of laptop ensembles: A survey of 160 laptop ensembles and their organisational structures’, in B. Caramiaux, K. Tahiroğlu, R. Fiebrink, and A. Tanaka (eds), Proceedings of the International Conference on New Interfaces for Musical Expression, London, 30 June - 4 July.

Kokotsaki, D. and Hallam, S. (2007), 'Higher education music students’ perceptions of the benefits of participative music making', Music Education Research, 9:1, pp. 93-109. 
Lebler, D. (2007), 'Student-as-master? Reflections on a learning innovation in popular music pedagogy’, International Journal of Music Education, 25:3, pp. 205-21.

Mudd, T. (2012), 'Developing transferable skills through engagement with higher education laptop ensembles’, Journal of Music, Technology \& Education, 5:1, pp. 29-41.

Nettl, B. (1974), ‘Thoughts on improvisation: A comparative approach’, Musical Quarterly, pp. 1-19.

NIME (2001) New Interfaces for Musical Expression, http://www.nime.org/. Accessed 29 January 2015.

O’Neill, S. A. (2012), 'Becoming a music learner: Towards a theory of transformative music engagement', in G. E. McPherson and G. F. Welch (eds), G. E. McPherson and G. F. Welch (eds), The Oxford Handbook of Music Education, Volume 1, Oxford: OUP, pp. 163-86.

Orr, S. (2010), 'Collaborating or fighting for the marks? Students' experiences of group work assessment in the creative arts', Assessment \& Evaluation in Higher Education, 35:3, pp. 301-13.

Pitts, S. E. (2010), 'Musical education as a social act: learning from and within musical communities', in J. Ballantyne and B. Bartleet (eds), Navigating Sound and Music Education, Newcastle-upon-Tyne: Cambridge Scholars 
Publishing, pp. 115-28.

Pure Data (2014), Pure Data, http://puredata.info/. Accessed 30 August 2014.

Rose, S. and MacDonald, R. (2012), 'Improvisation as real-time composition', in D. Collins (ed.), The Act of Musical Composition: Studies in the Creative Process, Farnham: Ashgate, pp. 187-213.

Salazar, D. (2010), 'Tackling the isolated learning experience and its potential to impede creative engagement with music technology at HE level’, paper presented at SEMPRE Researching Music, Technology and Education Conference, University of Hull, 18 September.

Savage, J. (2012), ‘Those who can, play; those who can’t, use Music Tech', in C. Philpott and G. Spruce (eds), Debates in Music Teaching, London: Routledge, pp. 169-84.

Smallwood, S., Trueman, D., Cook, P. R. and Wang, G. (2008), 'Composing for laptop orchestra’, Computer Music Journal, 32:1, pp. 9-25.

Snyder, J. (2015), PLOrk: The Princeton Laptop Orchestra, http://plork.cs.princeton.edu/. Accessed 28 August 2014.

Spruce, G. (2012), 'Musical knowledge, critical consciousness and critical thinking', in C. Philpott and G. Spruce (eds), Debates in Music Teaching, London: 
Routledge, pp. 185-96.

Tinto, V. (2003), 'Learning better together: The impact of learning communities on student success', Higher Education Monograph Series, 1:8, http://www.gvsu.edu/cms3/assets/B85DAC41-B7B8-3B9FA116121D5AE29B05/Learning\%20Better\%20Together.pdf. Accessed 30 August 2014.

Trueman, D. (2007), ‘Why a laptop orchestra?’, Organised Sound, 12:02, pp. 171-79.

Tsabary, E. (2014), 'Music education through innovation: The concordia laptop orchestra as a model for transformational education’, in L. Gómez Chova, A. López Martínez and I. Candel Torres (eds), INTED2014 Proceedings, Valencia, Spain: IATED, pp. 657-64.

Webb, M. and Seddon, F. A. (2012), 'Musical instrument learning, music ensembles, and musicianship in a global and digital age', in G. E. McPherson and G. F. Welch (eds), The Oxford Handbook of Music Education, 1, New York: Oxford University Press, pp. 752-68.

Weinberg, G. (2005), 'Interconnected musical networks: Toward a theoretical framework', Computer Music Journal, 29:2, pp. 23-39.

Wenger, E., McDermott, R. A., and Snyder, W. (2002), Cultivating Communities of Practice: A Guide to Managing Knowledge, Massachusetts: Harvard Business 
School.

Wiggins, J. (2007), 'Compositional process in music’, in L. Bresler (ed), International Handbook of Research in Arts Education, New York: Springer, pp. 453-76.

Wilson, G. B. and MacDonald, R. A. (2012), 'The sign of silence: Negotiating musical identities in an improvising ensemble’, Psychology of Music, 40:5, pp. $558-73$.

\section{Contributor details}

Oded Ben-Tal is a composer and researcher working at the intersection of music, computing and cognition. His composition include both acoustic pieces, works combining instruments with electronics and multimedia work. He is a senior lecturer at the Music Department, Kingston University where he teaches composition, electronic music, and music psychology.

Diana Salazar is a composer and sound artist. Her practice-led research examines spatial composition and interpretation in electronic music and associated issues of performance practice and cross-disciplinary discourse. Her output includes acousmatic work, work for instruments and electronics, cross-disciplinary collaborations, and improvised electronic laptop performance. She is a lecturer in music at City University London. 
Contact:

Music Department, Kingston University, Penrhyn Road, Kingston upon Thames, Surrey, KT1 2EE, UK.

E-mail: O.Ben-Tal@kingston.ac.uk

City University, Northampton Square, London. EC1V 0HB, UK.

E-mail: diana.salazar.1@city.ac.uk

Notes 
1 This also forms part of the MusDig research project at the University of Oxford, however at the time of writing the results had not yet been published.

2 Audio examples from Kudac concerts and rehearsals can be found at:

http://www.soundcloud.com/kudac, accessed 29 January 2015.

3 For examples of research in these areas see information on the New Interfaces for Musical

Expression (NIME) International Conferences from 2001 to present: http://www.nime.org/, accessed 29 January 2015.

4 It is interesting to note that the former category of 'orchestras' (PLOrk, SLOrk) were initiated and are directed by staff whose research interests were primarily in the engineering side of computer music while Kudac was founded by four composers engaging in practice-led music research.

Similarly, Plork, Slork, and similar ensembles include students from both music and science/engineering backgrounds. Kudac participants are mostly studying at the music department. This echoes Boehm's (2007) findings about the difference between music technology courses that emerged out of computing department to those that are housed in music departments. 